JouRnal of Universal Mathematics

Vol.3 No.2 PP.137-145 (2020)

ISSN-2618-5660

DOI: $10.33773 /$ jum. 829176

\title{
APPLICATION OF KHARRAT-TOMA TRANSFORM FOR SOLVING LINEAR VOLTERRA INTEGRAL EQUATIONS
}

\author{
NIHAN GÜNGÖR
}

\begin{abstract}
In this paper, a new integral transform, called Kharrat-Toma transform is used for solving convolution type linear Volterra integral equations of the first kind and also convolution type linear Volterra integral equation of the second kind. Some applications are given to explain the procedure of solution of linear Volterra integral equations using Kharrat-Toma transform.
\end{abstract}

\section{INTRODUCTION}

Integral equations have used for the solution of several problems in engineering, applied mathematics and mathematical physics since the 18th century. The integral equations have begun to enter the problems of engineering and other fields because of the relationship with differential equations which have wide range of applications and so their importance has increased in recent years. One can find relevant terminology related to integral equations in $[11,15,16,17]$.

The linear Volterra integral equation of the second kind has the form

$$
y(x)=f(x)+\lambda \int_{0}^{x} K(x, t) y(t) d t
$$

where $K(x, t)$ is the kernel of the equation and $\lambda$ is a parameter. Here $f(x)$ and $K(x, t)$ are specified functions but $y(x)$ is an unknown funciton that needs to be determined. The linear Volterra integral equation of the first kind is given by

$$
f(x)=\int_{0}^{x} K(x, t) y(t) d t .
$$

There are numerous integral transforms to solve integral equations, the most widely used of these transforms is Laplace transform. Asiru [7] demonstrated in solving convolution type integral equations by using Sumudu transform. Song and Kim [14] checked the Volterra integral equations of the second kind with an integral of the form of a convolution by using Elzaki transform. Haarsa [9] solved

Date: November, 2020.

2000 Mathematics Subject Classification. 45A05,45D05,44A99.

Key words and phrases. Integral equations, Integral transforms, Kharrat-Toma transform, Volterra integral equations. 
on Volterra integral equations of the first kind using Elzaki transform. Abdallah and Shama [1] solved the applications of differential transform method to integral equations. Aggarwal et al. [2, 3, 4, 5, 6] applied Aboodh, Kamal,Mahgoub and Shehu transforms for solving linear Volterra integral equations. Senthil Kumar et al. $[12,13]$ obtained the exact results of Mohand transforms for solving linear Volterra integro-differential equations and linear Volterra integral equations of first kind. Gnanavel et al. [8] applied Tarig transform for solving linear Volterra integral equations of first kind.

Kharrat and Toma [10] defined a new integral transform which is called KharratToma transform. They presented some properties of this transform and so they are provided a new mathematical tool to solve ordinary differential equation of variable and constant coefficients with initial conditions. The aim of this paper is to give exact solutions for convoluiton type linear Volterra integral equation of the first kind and also the second kind by using new integral transform:Kharrat-Toma transform without large computational work.

\section{Preliminaries}

In this section, we will give some necessary information about the new integral transform: Kharrat-Toma Transform.

Definition 2.1. If there exits a positive number $M$ that satisfying

$$
|f(x)| \leq M e^{\alpha x}, M>0, \alpha>0, \forall x \geq 0
$$

then the function $f(x)$ is said to have exponential order on every finite interval in $[0,+\infty)[10]$.

Definition 2.2. The Kharrat-Toma integral transform and inversion is defined by

$$
\begin{gathered}
B[f(x)]=G(S)=s^{3} \int_{0}^{\infty} f(x) e^{\frac{-x}{s^{2}}} d x, x \geq 0 . \\
f(x)=B^{-1}[G(S)]=B^{-1}\left[s^{3} \int_{0}^{\infty} f(x) e^{\frac{-x}{s^{2}}} d x\right] .
\end{gathered}
$$

The $B$ integral transform states that, if $f(x)$ is piecewise continuous on $[0,+\infty)$ and has exponential order. The $B^{-1}$ will be the inverse of the $B$ integral transform [10].

Kharrat-Toma transforms and inverse of Kharrat-Toma transforms of some functions are given the follows: 


\begin{tabular}{|l|l|}
\hline$f(x)$ & $B[f(x)]=G(s)$ \\
\hline 1 & $s^{5}$ \\
\hline$x$ & $s^{7}$ \\
\hline$x^{n}$ & $n ! s^{2 n+5}$ \\
\hline$e^{k x}$ & $\frac{s^{5}}{1-k s^{2}}$ \\
\hline $\sin (k x)$ & $\frac{k s^{7}}{1+k^{2} s^{4}}$ \\
\hline $\cos (k x)$ & $\frac{s^{5}}{1+k^{2} s^{4}}$ \\
\hline $\sinh (k x)$ & $\frac{k s^{7}}{1-k^{2} s^{4}}$ \\
\hline $\cos (k x)$ & $\frac{s^{5}}{1-k^{2} s^{4}}$ \\
\hline
\end{tabular}

\begin{tabular}{|l|l|}
\hline$G(s)$ & $f(x)=B^{-1}[G(S)]$ \\
\hline$s^{5}$ & 1 \\
\hline$s^{7}$ & $x$ \\
\hline$n ! s^{2 n+5}$ & $x^{n}$ \\
\hline$\frac{s^{5}}{1-k s^{2}}$ & $e^{k x}$ \\
\hline$\frac{k s^{7}}{1+k^{2} s^{4}}$ & $\sin (k x)$ \\
\hline$\frac{s^{5}}{1+k^{2} s^{4}}$ & $\cos (k x)$ \\
\hline$\frac{k s^{7}}{1-k^{2} s^{4}}$ & $\sinh (k x)$ \\
\hline$\frac{s^{5}}{1-k^{2} s^{4}}$ & $\cos (k x)$ \\
\hline
\end{tabular}

Kharrat-Toma Transforms of Some Standart Result

Inverse Kharrat-Toma Transforms of Some Standart Result

Theorem 2.3 (Linearity property). Let $B\left[f_{1}(x)\right]=G_{1}(s), B\left[f_{1}(x)\right]=G_{1}(s), \ldots$, $B\left[f_{n}(x)\right]=G_{n}(s)$ and the constants $c_{1}, c_{2}, \ldots, c_{n}$, then

$$
B\left[\sum_{k=1}^{n} c_{k} f_{k}(x)\right]=\sum_{k=1}^{n} c_{k} B\left[f_{k}(x)\right][10] .
$$

Theorem 2.4 (Convolution Theorem). Let $B[f(x)]=M(s), B[g(x)]=N(s)$, then

$$
B[f(x) * g(x)]=\frac{1}{s^{3}} M(s) N(s)[10] .
$$

Here, the convolution of two functions $f(x)$ and $g(x)$ is defined by $f(x) * g(x)=$ $\int_{0}^{x} f(x-t) g(t) d t$.

Theorem 2.5 (Kharrat-Toma transform of $x^{n} f(x), n \geq 1$ ). If $B[f(x)]=G(s)$, then

$$
\begin{aligned}
B[x f(x)] & =\frac{s^{3}}{2} \frac{d G(s)}{d s}-\frac{3}{2} s^{2} G(s) \\
B\left[x^{2} f(x)\right] & =\frac{s^{6}}{4} \frac{d^{2} G(s)}{d s^{2}}-\frac{3}{4} s^{5} \frac{d G(s)}{d s}+\frac{3}{4} s^{4} G(s)[10]
\end{aligned}
$$

\section{Kharrat-Toma Transform for Solving to Convolution Type Linear Volterra Integral Equations}

In this work, we deal with the kernel $K(x, t)$ as a convolution type kernel that can be expressed by the difference $(x-t)$. The convolution type linear Volterra integral equation of the second kind with the form of

$$
y(x)=f(x)+\lambda \int_{0}^{x} K(x-t) y(t) d t
$$

and the convolution type linear Volterra integral equation of the first kind with the form of

$$
f(x)=\int_{0}^{x} K(x-t) y(t) d t
$$


Theorem 3.1. The solution of Volterra integral equation of the first kind

$$
f(x)=\int_{0}^{x} K(x-t) y(t) d t
$$

is expressed by

$$
y(x)=B^{-1}[G(s)]=B^{-1}\left[\frac{s^{3} B[f(x)]}{B[K(x)]}\right]
$$

where $K$ is the kernel and $B[y(x)]=G(s)$

Proof. Let taking the Kharrat-Toma transform to both sides of VIEFK (3.1), we have

$$
\begin{aligned}
& B[f(x)]=B\left[\int_{0}^{x} K(x-t) y(t) d t\right] \\
& B[f(x)]=B[K(x) * y(x)] .
\end{aligned}
$$

Using convolution theorem of Kharrat-Toma transform, we find

$$
\begin{aligned}
B[f(x)] & =\frac{1}{s^{3}} B[K(x)] B[y(x)] \\
B[y(x)] & =\frac{s^{3} B[f(x)]}{B[K(x)]} .
\end{aligned}
$$

Operating inverse Kharrat-Toma transform on both sides of (3.2), we get

$$
y(x)=B^{-1}\left[\frac{s^{3} B[f(x)]}{B[K(x)]}\right]
$$

which is the required solution of (3.1).

Theorem 3.2. The solution of Volterra integral equation of the second kind

$$
y(x)=f(x)+\lambda \int_{0}^{x} K(x-t) y(t) d t
$$

is expressed by

$$
y(x)=B^{-1}[G(s)]=B^{-1}\left[\frac{B[f(x)]}{1-\frac{\lambda}{s^{3}} B[K(x)]}\right]
$$

where $K$ is the kernel and $B[y(x)]=G(s)$.

Proof. Applying the Kharrat-Toma transform to both sides of VIESK (3.3), we have

$$
\begin{aligned}
& B[y(x)]=B\left[f(x)+\lambda \int_{0}^{x} K(x-t) y(t) d t\right] \\
& B[y(x)]=B[f(x)]+\lambda B\left[\int_{0}^{x} K(x-t) y(t) d t\right] \\
& B[y(x)]=B[f(x)]+B[K(x) * y(x)] .
\end{aligned}
$$


Using convolution theorem of Kharrat-Toma transform, we find

$$
\begin{aligned}
B[y(x)] & =B[f(x)]+\frac{\lambda}{s^{3}} B[K(x)] B[y(x)] \\
B[y(x)] & =\frac{B[f(x)]}{1-\frac{\lambda}{s^{3}} B[K(x)]} .
\end{aligned}
$$

Operating inverse Kharrat-Toma transform on both sides of (3.4), we get

$$
y(x)=B^{-1}\left[\frac{B[f(x)]}{1-\frac{\lambda}{s^{3}} B[K(x)]}\right]
$$

which is the required solution of (3.3).

\section{APPLICATIONS}

In this section, some applications are given to explain the procedure of the solution of convolution type linear Volterra integral equations using Kharrat-Toma transform.

Example 4.1. Solve the following convolution type Volterra integral equation of the first kind

$$
x^{2}=\int_{0}^{x} e^{(x-t)} y(t) d t
$$

by using Kharrat-Toma transform method.

Let us write $B[y(x)]=G(s)$. Apply the Kharrat-Toma transform

$$
B\left[x^{2}\right]=B\left[\int_{0}^{x} e^{(x-t)} y(t) d t\right]
$$

Using convolution theorem of above integral equation, we write

$$
\begin{aligned}
s^{9} .2 ! & =\frac{1}{s^{3}} B\left[e^{x}\right] B[y(x)] \\
2 s^{9} & =\frac{1}{s^{3}} \frac{s^{5}}{1-s^{2}} G(s) .
\end{aligned}
$$

Hence, we find

$$
B[y(x)]=G(s)=2 s^{7}\left(1-s^{2}\right) .
$$

Operating inverse Kharrat-Toma transform, we get

$$
y(x)=B^{-1}\left[2 s^{7}-2 s^{9}\right]=2 x-x^{2} .
$$

Therefore, we have the answer

$$
y(x)=2 x-x^{2} .
$$

Example 4.2. Solve the following convolution type Volterra integral equation of the second kind

$$
y(x)=\cos x-\int_{0}^{x}(x-t) \cos (x-t) y(t) d t
$$


by using Kharrat-Toma transform method.

Let us write $B[y(x)]=G(s)$. Let applying the Kharrat-Toma transform

$$
\begin{aligned}
B[y(x)] & =B\left[\cos x-\int_{0}^{x}(x-t) \cos (x-t) y(t) d t\right] \\
& =B[\cos x]-B\left[\int_{0}^{x}(x-t) \cos (x-t) y(t) d t\right] \\
& =B[\cos x]-B[x \cos (x) * y(x)]
\end{aligned}
$$

Using convolution theorem of above integral equation, we have

$$
\begin{aligned}
G(s) & =B[\cos x]-\frac{1}{s^{3}} B[x \cos (x)] B[y(x)] \\
& =\frac{s^{5}}{1+s^{4}}-\frac{1}{s^{3}}\left[\frac{s^{3}}{2} \frac{5 s^{4}+s^{8}}{\left(1+s^{4}\right)^{2}}-\frac{3}{2} s^{2} \frac{s^{5}}{1+s^{4}}\right] G(s) \\
& =\frac{s^{5}}{1+s^{4}}-\frac{1}{s^{3}}\left[\frac{s^{7}-s^{11}}{\left(1+s^{4}\right)^{2}}\right] G(s) \\
& =\frac{s^{5}}{1+s^{4}}-\left[\frac{s^{4}-s^{8}}{\left(1+s^{4}\right)^{2}}\right] G(s)
\end{aligned}
$$

Hence, we find

$$
\begin{aligned}
B[y(x)] & =G(s)=\frac{s^{5}\left(1+s^{4}\right)^{2}}{\left(3 s^{4}+1\right)\left(1+s^{4}\right)}=\frac{s^{9}+s^{5}}{1+3 s^{4}} \\
& =\frac{1}{3} s^{5}-\frac{1}{3} \frac{s^{5}}{1+3 s^{4}}+\frac{s^{5}}{1+3 s^{4}} \\
& =\frac{1}{3} s^{5}+\frac{2}{3} \frac{s^{5}}{1+3 s^{4}} .
\end{aligned}
$$

Operating inverse Kharrat-Toma transform, we get

$$
\begin{aligned}
y(x) & =B^{-1}\left[\frac{1}{3} s^{5}+\frac{2}{3} \frac{s^{5}}{1+3 s^{4}}\right] \\
& =\frac{1}{3} B^{-1}\left[s^{5}\right]+\frac{2}{3} B^{-1}\left[\frac{s^{5}}{1+3 s^{4}}\right]+ \\
& =\frac{1}{3}+\frac{2}{3} \cos (\sqrt{3} x) .
\end{aligned}
$$

Therefore, we have the answer

$$
y(x)=\frac{1}{3}+\frac{2}{3} \cos (\sqrt{3} x) .
$$

Example 4.3. Solve the following convolution type Volterra integral equation of the second kind

$$
y(x)=\sin x+2 \int_{0}^{x} \cos (x-t) y(t) d t
$$


by using Kharrat-Toma transform method.

Let us write $B[y(x)]=G(s)$. Let applying the Kharrat-Toma transform

$$
\begin{aligned}
B[y(x)] & =B\left[\sin x+2 \int_{0}^{x} \cos (x-t) y(t) d t\right] \\
& =B[\sin x]+2 B\left[\int_{0}^{x} \cos (x-t) y(t) d t\right] \\
& =B[\sin x]+2 B[\cos x * y(x)]
\end{aligned}
$$

Using convolution theorem of above integral equation, we have

$$
\begin{aligned}
G(s) & =B[\sin x]+2 \frac{1}{s^{3}} B[\cos x] B[y(x)] \\
& =\frac{s^{7}}{1+s^{4}}+2 \frac{1}{s^{3}} \frac{s^{5}}{1+s^{4}} G(s) \\
& =\frac{s^{7}}{1+s^{4}}+2 \frac{s^{2}}{1+s^{4}} G(s)
\end{aligned}
$$

Hence, we find

$$
B[y(x)]=G(s)=\frac{s^{7}}{\left(1-s^{2}\right)^{2}} .
$$

Operating inverse Kharrat-Toma transform, we get

$$
y(x)=B^{-1}\left[\frac{s^{7}}{\left(1-s^{2}\right)^{2}}\right]=x e^{x} .
$$

Therefore, we have the answer

$$
y(x)=x e^{x} .
$$

Example 4.4. Let us consider the initial value problem

$$
\left\{\begin{array}{c}
y^{\prime \prime}(x)-2 y^{\prime}(x)-3 y(x)=0 \\
y(0)=1, y^{\prime}(0)=2 .
\end{array}\right.
$$

This equation is equivalent to

$$
y(x)=1+\int_{0}^{x}[3(x-t)+2] y(t) d t
$$

Volterra integral equation. We can write the equation as

$$
y(x)=1+3 \int_{0}^{x}(x-t) y(t) d t+2 \int_{0}^{x} y(t) d t .
$$

If we take Kharrat-Toma transform on both sides, we obtain

$$
\begin{aligned}
B[y(x)] & =B[1]+3 B\left[\int_{0}^{x}(x-t) y(t) d t\right]+2 B\left[\int_{0}^{x} y(t) d t\right] \\
& =B[1]+3 \frac{1}{s^{3}} B[x * y(x)]+2 \frac{1}{s^{3}} B[1 * y(x)] .
\end{aligned}
$$


Let us write $B[y(x)]=G(s)$ and using convolution theorem of above integral equation, we have

$$
\begin{aligned}
G(s) & =s^{5}+3 \frac{1}{s^{3}} B[x] B[y(x)]+2 \frac{1}{s^{3}} B[1] B[y(x)] \\
& =s^{5}+3 s^{4} G(s)+2 s^{2} G(s) .
\end{aligned}
$$

Hence, we find

$$
B[y(x)]=G(s)=\frac{s^{5}}{1-2 s^{2}-3 s^{4}}=\frac{s^{5}}{\left(1+s^{2}\right)\left(1-3 s^{2}\right)}=\frac{1}{4} \frac{s^{5}}{1+s^{2}}+\frac{3}{4} \frac{s^{5}}{1-3 s^{2}} .
$$

Operating inverse Kharrat-Toma transform, we get

$$
\begin{aligned}
y(x) & =B^{-1}\left[\frac{1}{4} \frac{s^{5}}{1+s^{2}}+\frac{3}{4} \frac{s^{5}}{1-3 s^{2}}\right] \\
& =\frac{1}{4} B^{-1}\left[\frac{s^{5}}{1+s^{2}}\right]+\frac{3}{4} B^{-1}\left[\frac{s^{5}}{1-3 s^{2}}\right] \\
& =\frac{1}{4} e^{-x}+\frac{3}{4} e^{3 x}
\end{aligned}
$$

Therefore, we have the answer

$$
y(x)=\frac{1}{4} e^{-x}+\frac{3}{4} e^{3 x}
$$

\section{CONCLUSION}

In this paper, we apply the new integral transform Kharrat-Toma transform to find the exact solution of convolution type linear Volterra integral equation of first and also second kind. The given applications are showed the exact solutions of integral equations that have been obtained by very less computational work and spending a very little time. Also, the solution of some ordinary differential equations with constant coefficients are found by Kharrat-Toma transform after converting them to Volterra integral equations.

\section{REFERENCES}

[1] H. A. Abdallah and A. M. Shama, Applications of differential transform method to integral equations, Amer. J. Engg. Res. (AJER), vol.7(1), pp.71-276 (2018).

[2] S. Aggarwal, R. Chauhan and N. Sharma, Application of Aboodh transform for solving linear Volterra integro-differential equations of second kind, Int. J. Res. Adv. Tech., vol.6(8), pp. 1186-1190 (2018).

[3] S. Aggarwal, R. Chauhan and N. Sharma, A new application of Kamal transform for solving linear Volterra integral equations, Int. J. Lat. Tech. Engg., Magn. \&App. Sci., vol.7(4), pp.138140 (2018).

[4] S. Aggarwal, R. Chauhan and N. Sharma, Application of Kamal transform for solving linear Volterra integral equations of first kind, Int. J. Res. Adv. Tech., vol.6(8), pp.2081-2088 (2018).

[5] S. Aggarwal, R. Chauhan and N. Sharma, A new application of Mahgoub transform for solving linear Volterra integral equations, Asi. Res., Vol.7(2), pp. 46-48 (2018).

[6] S. Aggarwal, R. Chauhan and N. Sharma, A New Application of Shehu Transform for Handling Volterra Integral Equations of First Kind, International Journal of Research in Advent Technology, Vol.7(4), pp.439-445 (2019).

[7] M. A. Asiru, Sumudu Transform and the Solution of Integral Equations of Convolution Type, International Journal of Mathematical Education in Science and Technology, vol.32(6), pp.906-910 (2001). 
[8] M.G. Gnanavel, C. Saranya and A. Viswanathan, Applications of Linear Volterra Integral Equations of First Kind by Using Tarig Transform, International Journal of Innovative Technology and Exploring Engineering (IJITEE), Vol.8(10), pp.2278-3075 (2019).

[9] P. Haarsa, On Volterra integral equations of the first kind by using Elzaki transform," Far East J. Mat. Sci. (FJMS), vol. 102(9), pp.1857-1863 (2017).

[10] B.N.Kharrat and G.A.Toma, A New Integral Transform:Kharrat-Toma Transform and Its Properties, World Applied Sciences Journal, vol.38(5), pp.436-443 (2020).

[11] M. Rahman, Integral Equations and Their Applications, WIT press, Boston, (2007).

[12] P. Senthil Kumar, M. G. Gnanavel and V. Viswanathan, Application of Mohand Tranform for Solving Linear Volterra Integro - Differential Equations", Int. J. Res. Adv. Tech., vol. 6(10), pp. 2554-2556 (2018).

[13] P. Senthil Kumar, C. Saranya, M. G. Gnanavel and A. Viswanathan, Application of Mohand Tranform for Solving Linear Volterra Integral Equations of First Kind, Int. J. Res. Adv. Tech., vol. 6(10), pp. 2786-2789 (2018).

[14] Y. Song and H. Kim, The Solution of Volterra Integral Equation of the Second Kind by Using the Elzaki Transform, Applied Mathematical Sciences, Vol.8(11), pp.525-530 (2014).

[15] V. Volterra, Theory of functional of integral and integro-differential equations, Dover, New York, (1959).

[16] A. M. Wazwaz, A first course in integral equations. World Scientific, Second Edition, Singapore, (2015).

[17] A. M. Wazwaz, Linear and Nonlinear Integral Equations Methods and Applications, Springer Verlag Berlin Heidelberg, (2011).

Gumushane University, Department of Mathematical Engineering, 29100, Gumushane, TURKEY

Email address: nihangungor@gumushane.edu.tr 